\title{
Thrombelastography Parameters versus Classical Coagulation Profile in Trauma Patients: a Retrospective Study
}

\author{
Abdulraouf Y. Lamoshi \\ West Virginia University
}

Follow this and additional works at: https://researchrepository.wvu.edu/etd

\section{Recommended Citation}

Lamoshi, Abdulraouf Y., "Thrombelastography Parameters versus Classical Coagulation Profile in Trauma Patients: a Retrospective Study" (2013). Graduate Theses, Dissertations, and Problem Reports. 4977. https://researchrepository.wvu.edu/etd/4977

This Thesis is protected by copyright and/or related rights. It has been brought to you by the The Research Repository @ WVU with permission from the rights-holder(s). You are free to use this Thesis in any way that is permitted by the copyright and related rights legislation that applies to your use. For other uses you must obtain permission from the rights-holder(s) directly, unless additional rights are indicated by a Creative Commons license in the record and/ or on the work itself. This Thesis has been accepted for inclusion in WVU Graduate Theses, Dissertations, and Problem Reports collection by an authorized administrator of The Research Repository @ WVU. For more information, please contact researchrepository@mail.wvu.edu. 


\title{
Thrombelastography Parameters versus Classical Coagulation Profile in Trauma Patients: a Retrospective Study
}

\author{
by \\ Abdulraouf Y. Lamoshi \\ Thesis submitted to the \\ School of Medicine \\ at West Virginia University \\ in partial fulfillment of the requirements \\ for the degree of
}

Master in Clinical and Translational Sciences

in

Fawad Khan, MBBS

Christa L. Ice, PhD

Alison Wilson, MD, FACS, Chair

Morgantown, West Virginia

2013

Keywords: Thrombelastography, Traumatic Brain Injury, Classical Coagultion

Copyright 2013 Abdulraouf Y. Lamoshi 


\begin{abstract}
Thrombelastography Parameters versus Classical Coagulation Profile in Trauma Patients: a Retrospective Study

by

Abdulraouf Y. Lamoshi

Master in Clinical and Translational Sciences in

West Virginia University

Alison Wilson, MD, FACS, Chair
\end{abstract}

The West Virginia University Guide to the Preparation of Masters Theses Abdulraouf Y. Lamoshi Thrombelastography Parameters versus Classical Coagulation Profile in Trauma Patients: a Retrospective Study Background: Thrombelastography (TEGTM, Hemoscope, Niles, IL) is increasingly utilized. As TEG depicts overall coagulation it may be more comprehensive and capable to detect any coagulation abnormalities in comparison to classical coagulation tests (CCT: PT, PTT, INR, platelets). Traumatic brain injury (TBI) is thought to contribute to coagulopathy. The primary aim was to compare TEG parameters of TBI vs. Non-TBI patients. The secondary aim was to identify TEG vs. CCT parameters associated with outcome (mortality, need for transfusion, length of stay).

Methods: This is a cross sectional retrospective observational study of 142 patients (full trauma team activations only) admitted to a university based, Level 1 trauma center. TEG and CCT were collected on admission. Citrated Kaolin samples were utilized. Data was analyzed by a biostatistician using JMP V10.

Results: 142 patients (pts), 44 (31\%) women and 98 (69\%) men. 48 pts had TBI and 94 pts were NTBI. Overall mortality was $20.4 \%$ (45.8\% TBI vs. $7.4 \%$ NTBI). There were no significant associations between TEG or CCT parameters and ISS, scene vs. transfer, hospital LOS, or ventilator days. There was no difference between the TBI and NTBI groups in terms of TEG or CCT parameters. Variables found to be associated with mortality were K $(p=0.0118)$ and age $(p=0.0057)$. MA was the only parameter (TEG or CCT) associated with need for transfusion of PRBC $(\mathrm{p}=0.0377)$. PRBC transfusion was given in $94 \%$ of 16 patients with an $M A \leq 57.4$ (1-4 units in $44 \%$ and $\geq 4$ units in $50 \%$ ). Platelet transfusion was given in $89 \%$ of 9 patients who have $M A \leq 58.1$. FFP transfusion was given in $80 \%$ of 15 patients who have $R \geq 5.8$. Decreased MA $(p=0.0003), \downarrow \mathrm{K}(p=0.0154)$, $\uparrow \mathrm{PT}$ $(p=0.0015)$, and $\uparrow \operatorname{INR}(p=0.0014)$ are significantly associated with FFP transfusion. K value is significantly associated with mortality $(p=0.0118)$ and hypotension $(p=0.0172)$. Conclusions: TEG parameters are potentially useful as means to rapidly diagnose coagulopathy and predict transfusion in trauma pts. Independently, presence of TBI does not cause a detectable coagulopathy. MA is best single indicator for PRBC and/or FFP transfusion in trauma patients. TEG analysis is more efficient than the classical parameters in detecting patients who will need PRBC and FFP transfusion. 


\section{Acknowledgements}

I owe my deepest gratitude and thanks to my mentor, Dr. Wilson, for giving me the opportunity to work with her. This thesis would not be possible without her guidance and support. Also it is an honor for me to thank Dr. Khan and Dr. Lilly for being on my committee. The opportunity to take courses with Dr. Lilly, gave me essential understanding of the biostatistics perspective and Dr. Khan for his clinical invaluable clinical feedback. Next, I would like to thank West Virginia Clinical and Translational Sciences Institute (WVCTSI) for offering biostatistics service. Finally, I would also like to express my attitude to my wife and daughter for their priceless psychological support. 


\section{Contents}

$\begin{array}{ll}\text { Acknowledgements } & \text { iii }\end{array}$

$\begin{array}{ll}\text { List of Figures } & \text { v }\end{array}$

1 Background 1

2 Methods and Results 5

2.1 Methods . . . . . . . . . . . . . . . . . . . . . 5

2.2 Results . . . . . . . . . . . . . . . . . . . . 6

3 Discussion $\quad 12$

$\begin{array}{ll}\text { References } & 16\end{array}$ 


\section{List of Figures}

1.1 An overview of the cell-based model of coagulation. Curry, Andy NG, and JM Tom Pierce. "Conventional and near-patient tests of coagulation." Continuing Education in Anaesthesia, Critical Care \& Pain 7, no. 2 (2007): 45-50. . . .

1.2 : Graphical Representation of TEG Parameters. Ereth, Mark. Blood CME Center, "Uncontrolled Bleeding After Thoracic Aortic Aneurysm Repair: A Case Report and Interactive Discussion." Last modified 2013. Accessed June 10, 2013. http://www.bloodcmecenter.org/BeginActivity.aspx?AID $=49 \& \mathrm{ST}=1$ $\& \mathrm{pg}=$ ClinicalBrief1/Intro. . . . . . . . . . . . . . . 3

2.1 Correlation of MA Cut-off Point with PRBC Transfusion . . . . . . . . . . 8

2.2 Correlation of MA Cut-off Point with Platelets Transfusion . . . . . . . . . . 8

2.3 Correlation of R Cut-off Point with FFP Transfusion . . . . . . . . . . . . 9

2.4 Correlation of Alpha Cut-off Point with FFP Transfusion . . . . . . . . . . . 9

2.5 Correlation of K Measurement with Blood Pressure Status . . . . . . . . . . 10

2.6 Correlation of K Measurement with Mortality Rate . . . . . . . . . . . . . . 10

2.7 Correlation of K Measurement with FFP Transfusion . . . . . . . . . . . . . 11 


\section{Chapter 1}

\section{Background}

Blood transfusion protocols are crucial measures in all trauma settings and many other medical situations. Choosing the appropriate timing and type of blood and/or blood product transfusions are sometimes vital decisions that determine the future of trauma patients [1] Head injury forms approximately $45 \%$ of trauma-related mortalities and bleeding is the second most common cause of death, 30\%, during the early stages of trauma[2] On the other hand, multiorgan failure leads to $8 \%$ of deaths in the late trauma incidents, which is associated with massive transfusion that can lead to coagulopathy in $65 \%$ of the cases and increase the risk of death by $50 \%[2]$. Thus, constructing a scientifically founded strategy to predict the precise need for transfusion to face the different scenarios can save a lot of morbidities and fatalities. In 1948 Hartert invented the Thromboelastograph (TEG) technique to quantify the dynamics of clot development and termination throughout its progression stages where it evaluates the interaction between fibrinogen, platelets, and clotting factors. 3 In comparison, the classical laboratory screening tests of coagulation, which include fibrinogen level, thrombin clotting times (TCT or thrombin time, TT), the PT, and the APTT, measure only the progression of the clotting cascade until the time of first fibrin development (Figure 1.1).3 Moreover, aPTT and INR neglect the platelets effect because the tests are executed on platelet-poor plasma samples and CCT results may take up to 30 minutes to be available which means precious time for intervention is lost 4.5. The main TEG parameters are R-time (or reaction time), K-time (or clot formation time), angle of alpha $(\alpha)$ (function of rate of fibrin polymerization) and MA (Maximum Amplitude) (Figure 1.2).3 
$\mathrm{R}$ is increased due to anticoagulants, factor deficiencies, and severe hypofibrinogenaemia

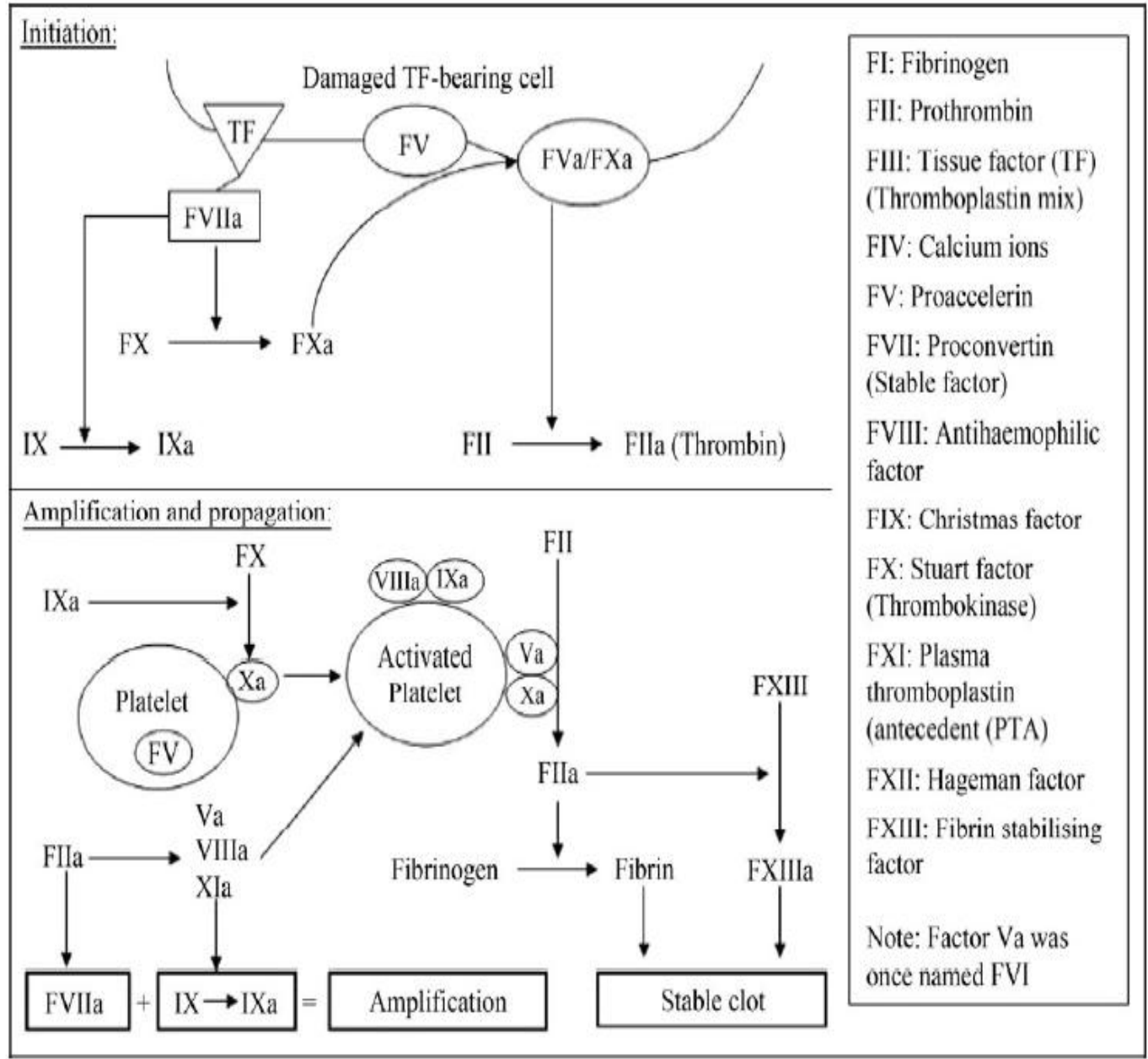

Figure 1.1: An overview of the cell-based model of coagulation. Curry, Andy NG, and JM Tom Pierce. "Conventional and near-patient tests of coagulation." Continuing Education in Anaesthesia, Critical Care \& Pain 7, no. 2 (2007): 45-50.

and decreased by hypercoagulable conditions.3,6 $\mathrm{K}$ is increased due to anticoagulants, hypofibrinogenaemia, thrombocytopenia and declined by increased fibrinogen level, increased platelet function.3.6 $\alpha$ is prolonged by increased fibrinogen level, increased platelet function and shortened by anticoagulants, hypofibrinogenaemia, thrombocytopenia.3,6 MA is prolonged by hypercoagulable states, thrombocytosis and shortened by thrombocytopenia, platelet blockers, fibrinolysis, factor deficiencies.3,6 


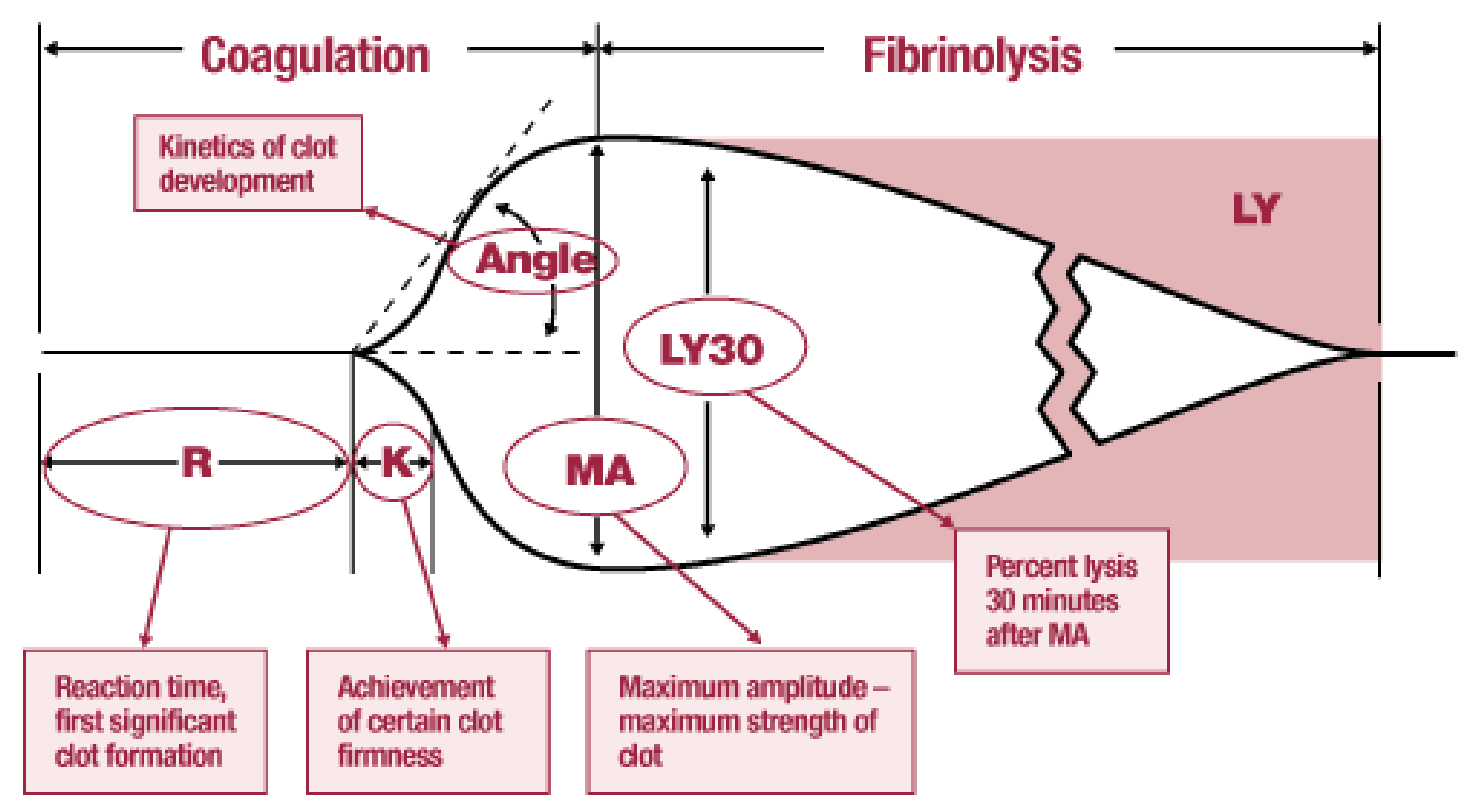

Figure 1.2: : Graphical Representation of TEG Parameters. Ereth, Mark. Blood CME Center, "Uncontrolled Bleeding After Thoracic Aortic Aneurysm Repair: A Case Report and Interactive Discussion." Last modified 2013. Accessed June 10, 2013. http://www.bloodcmecenter.org/BeginActivity.aspx?AID=49 \& ST=1 \& $\mathrm{pg}=$ ClinicalBrief1/Intro.

Severely injured patients may need large amounts of blood and blood products; however, there is no accurate blood tests that can predict the optimum time, type, and amount that patients really need[3] Depending primarily on the classical coagulation lab tests has many limitations that may lead to deadly outcomes[3] Post-traumatic patients, who are in hypovolemia shock, may need massive transfusion which causes dilution of coagulation factors and platelets that in turn leads to coagulopathy disorders 7 including venothromboembolic consequences[4]. TEG has been used to predict the demand for blood transfusion in surgeries that have high likelihood of bleeding settings such as orthotopic surgeries, liver transplantation and cardiopulmonary bypass, and in the field of trauma where it showed precise prediction for transfusion in the first day[4]. Nonetheless, TEG has not been used to measure the severity of coagulability based on different types of trauma, for example, traumatic brain injury[4]. According to previous studies, coagulopathy is more likely to develop in brain traumatic injuries patients and correlates to their high death rates[5][6]. Although the exact 
correlation between TBI and DIC is not fully explained, as a consequence to TBI, vascular or tissue injury releases a brain rich tissue factor (thromboplastin), which stimulates other extrinsic pathway procoagulant proteins that traps platelets and activates further thrombosis including DIC[6][7][8]. Moreover, TBI inhibits $\alpha$ 2-plasmin inhibitor activity which leads to major fibrinogenolysis[6].

The primary goal of this retrospective observational study was to compare TEG parameters of TBI vs. NTBI patients. The secondary aim was to identify TEG vs. CCT parameters association with the studied outcomes, including prediction of transfusion needs in trauma. 


\section{Chapter 2}

\section{Methods and Results}

\subsection{Methods}

This is a cross-sectional retrospective observational study of 142 patients (full trauma team activations only) admitted to a university based, Level 1 trauma center. TEG and CCT (INR, aPTT, TT, fibrinogen, platelet count) were collected on admission. Citrated Kaolin samples were utilized. TEG machines are subjected to electronic controls three times daily and liquid controls once a day. In addition to TEG and CCT values the following variables were collected: gender, age, scene versus transferred patients, ISS, AIS, Craniotomy/ectomy, type of brain injury, discharge status (alive versus dead), BP, blood and blood product transfusion, hospital length of stay, and ventilator days. The relationship between TEG and CCT parameters were analyzed using all these variables. TEG samples were sent from the trauma-bay within 15 minutes of arrival to the hospital. TBI cases diagnosed based on Glascow Coma Scale and CT scan which showed intracranial injury.

Statistical analysis was performed using SAS, version 10 (SAS Institute Inc, Cary, North Carolina). Categorical data were analyzed using Chi-square or Fisher exact test. A comparison of continuous variables between TBI and NTBI patients was performed using the independent-sample t test. Comparisons among the two groups were performed by one-way ANOVA followed by the Tukey correction for post-hoc comparisons. Wilcoxon / KruskalWallis Tests were used when appropriate. Pearson correlation coefficients were calculated between continuous variables. $\mathrm{P}$ values of.05 or less than were considered statistically sig- 
nificant and data are reported as mean (SEM).

\subsection{Results}

Out of 142 patients, there were 44 (31\%) women and 98 (69\%) men. Forty-eight patients had a TBI and 94 pts were NTBI. Overall mortality was $20.4 \%$ (45.8\% TBI vs $7.4 \%$ NTBI). Of the 48 TBI patients, 7 (15\%) were diagnosed with IVH, 22 (46\%) IPH/CC, [2] (4\%) EDH, 31 (64\%) SDH, and 26 (54\%) SAH. The patients with systolic blood pressure above 90 had a greater hospital length of stay (6.9 days vs. 14.7 days) $(P=.0001)$. Also SBP $\geq 90$ significantly associated with ISS where those patients who had low ISS (average of 13) had also SBP $\geq 90$ and those who had high ISS (average of 19) had also SBP $\leq 90$ $(P=003)$. There were no significant associations between TEG or CCT parameters and ISS, scene vs. transfer, hospital LOS, or ventilator days (Table 1). There was no difference between the TBI and NTBI groups in terms of TEG or CCT parameters (Table 2). The number of patients who were sent from the scene directly to our facility was 89 out of which $15(17 \%)$ died during the admission. Comparably, 53 patients were transferred from other facilities out of which $14(26 \%)$ died showing no significant difference in overall mortality $(P=.171)$. This matched the severity of injuries where $38 \%$ of the scene group had ISS greater than 15 compared with $34 \%$ of the transferred group $(P=.611)$. Variables found to be associated with mortality were $K(p=0.0118)$ and age $(p=0.0057)$.

MA was the only parameter (TEG or CCT) associated with the need for transfusion of $\operatorname{PRBC}(p=0.0377)$. PRBC transfusion was given in $94 \%$ of 16 patients with $\mathrm{MA} \leq 57.4$ (1-4 units in $44 \%$ and $\geq 4$ units in $50 \%$ ) and only in $53 \%$ of 63 patients with MA $\geq 57.4$ (Figure 2.1). MA also significantly associated with FFP transfusion (Figure 2.2).Platelet transfusion was given to $89 \%$ of 9 patients who have MA $\leq 58.1$ and only in $44 \%$ of 55 patients with $\mathrm{MA} \geq 58.1$. 
Table.1: P Value of Associations of all TEG and CCT Parameters with the Studied Variables

\begin{tabular}{|l|l|l|l|l|l|l|}
\hline Variable & $\mathrm{R}$ & $\mathrm{K}$ & $\mathrm{MA}$ & Angle & INR & PT \\
\hline Gender & 0.398 & 0.147 & 0.106 & & & \\
\hline Age & 0.343 & 0.895 & 0.148 & & & \\
\hline Length of stay & 0.297 & 0.736 & 0.830 & & 0.39 & 0.779 \\
\hline Ventilation days & 0.811 & 0.788 & 0.901 & & 0.207 & 0.192 \\
\hline Scene vs. transfer & 0.868 & 0.971 & 0.086 & & 0.733 & 0.414 \\
\hline Mortality & 0.976 & 0.012 & 0.417 & 0.147 & & \\
\hline ISS $\leq 15$ & 0.137 & 0.562 & 0.078 & & & \\
\hline ISS & 0.476 & 0.138 & 0.254 & 0.832 & & \\
\hline SBP $\geq 90$ & 0.443 & 0.196 & 0.168 & & 0.857 & 0.141 \\
\hline Hypotension & 0.63 & 0.017 & 0.335 & 0.69 & & \\
\hline Coumadin & 0.64 & 0.628 & 0.383 & & 0.002 & \\
\hline PRBC & 0.31 & 0.284 & 0.038 & 0.113 & 0.7 & 0.663 \\
\hline FFP & 0.201 & 0.015 & 0.000 & 0.095 & 0.001 & 0.002 \\
\hline Platelets & 0.507 & 0.134 & 0.138 & 0.352 & 0.502 & 0.494 \\
\hline Craniotomy & 0.768 & 0.805 & 0.65 & 0.261 & & \\
\hline
\end{tabular}

Table.2: CCT and TEG Parameters of TBI vs. NTBI Patients

\begin{tabular}{|l|l|l||l|}
\hline Parameter & TBI $(\mathrm{X}+\mathrm{SE})$ & $\mathrm{NTBI}(\mathrm{X}+\mathrm{SE})$ & $\mathrm{P}$ value \\
\hline $\mathrm{R}$ & $9.21 \pm 2.73$ & $5.38 \pm 1.72$ & 0.237 \\
\hline K & $2.11 \pm 0.25$ & $1.63 \pm 1.6$ & 0.111 \\
\hline MA & $63.00 \pm 1.15$ & $63.04 \pm 0.72$ & 0.977 \\
\hline Angle & $67.11 \pm 1,33$ & $67.68 \pm 0.9$ & 0.726 \\
\hline INR & $1.25 \pm 0.14$ & $1.38 \pm 0.11$ & 0.518 \\
\hline PT & $12.77 \pm 0.55$ & $12.68 \pm 0.42$ & 0.91 \\
\hline
\end{tabular}




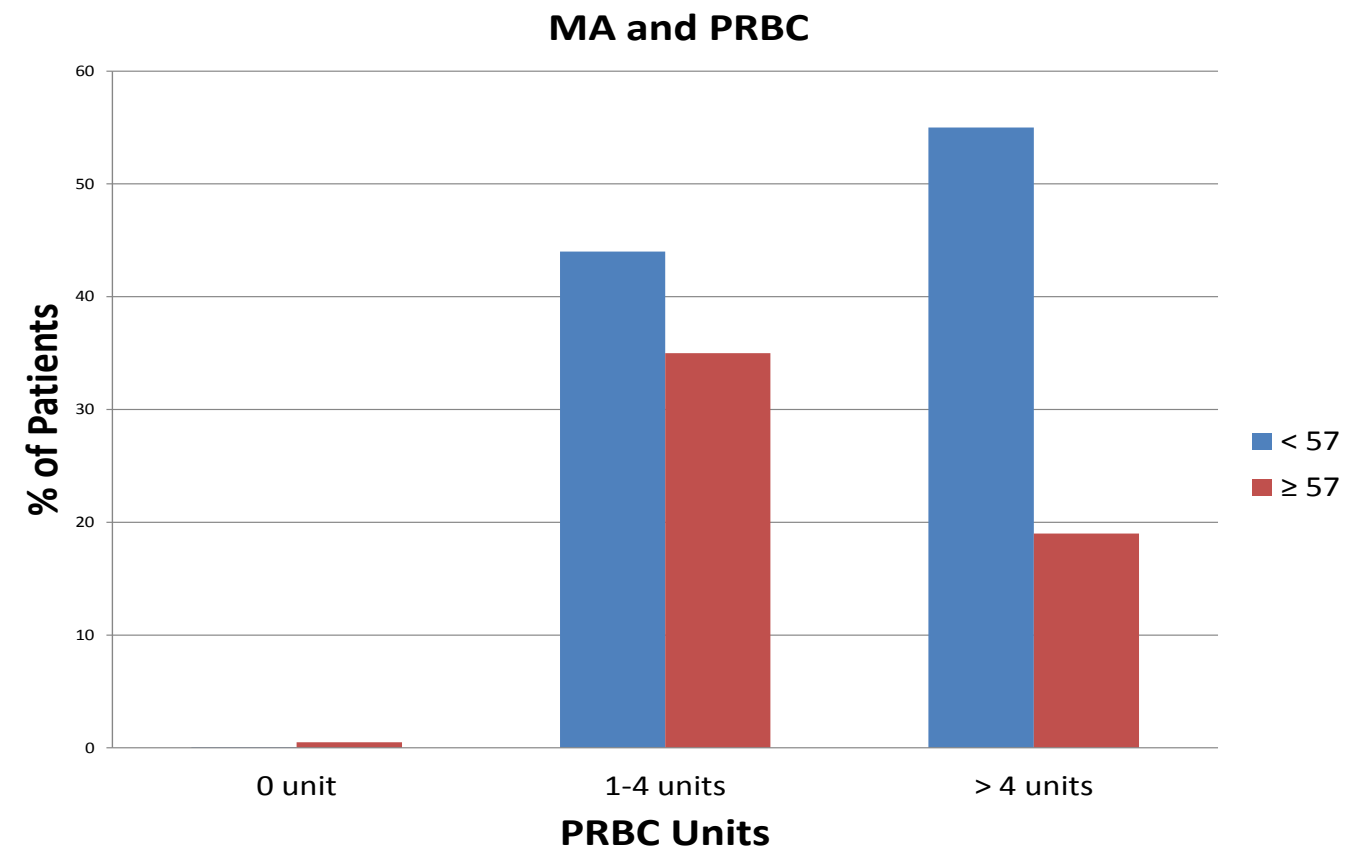

Figure 2.1: Correlation of MA Cut-off Point with PRBC Transfusion

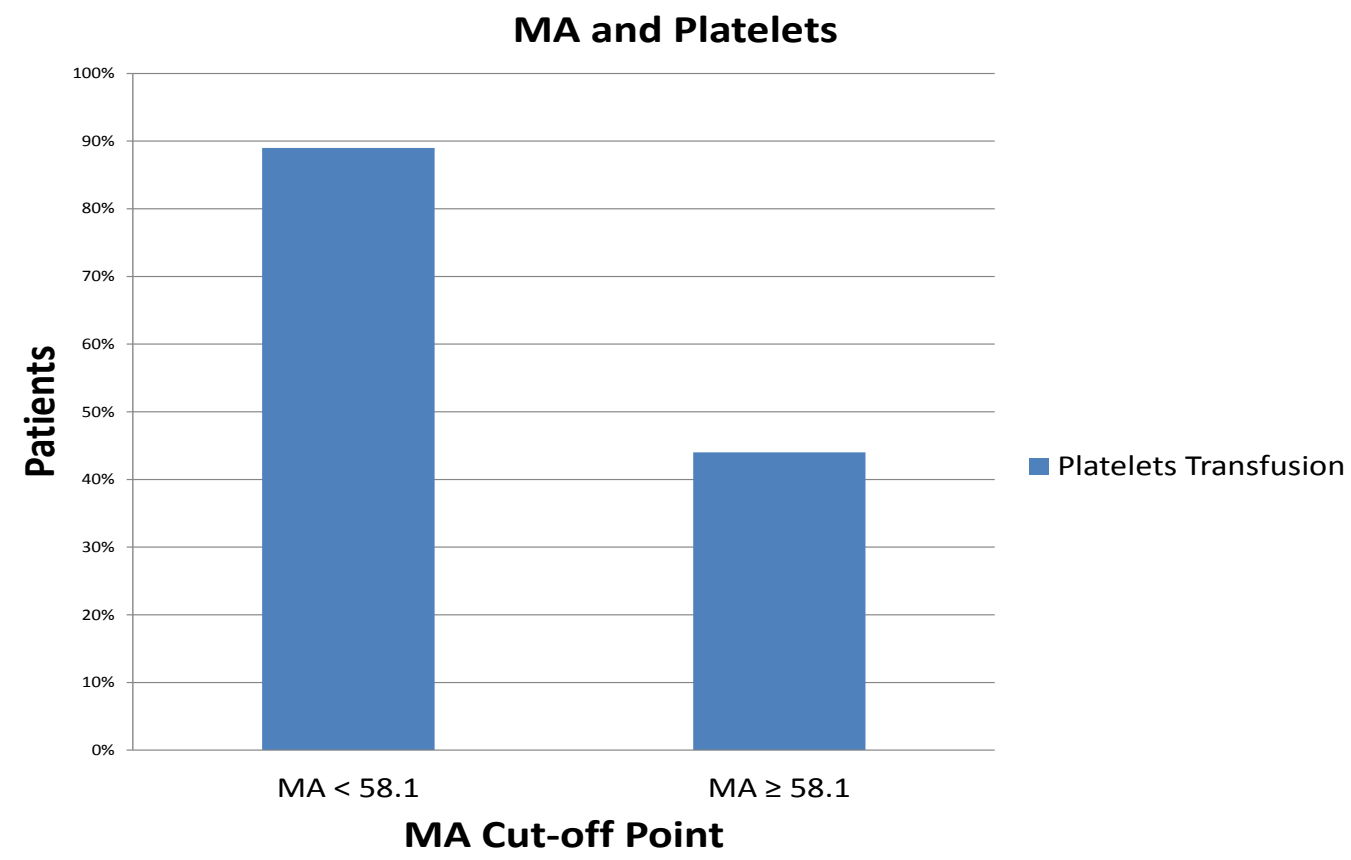

Figure 2.2: Correlation of MA Cut-off Point with Platelets Transfusion

Additionally, FFP transfusion was given to $80 \%$ of 15 patients who have $\mathrm{R} \geq 5.8$ and only in $32 \%$ of 50 patients with $\mathrm{R} \leq 5.8$ (Figure 2.3 ). with $\alpha \geq 62.9$. Platelet transfusion 
was given to $70 \%$ of 14 patients who have $\alpha \leq 64.5$ and only in $44 \%$ of 50 patients with $\alpha \geq$ 64.5. FFP transfusion was given to $67 \%$ of 25 patients who have $\alpha \leq 66.3$ and only in $28 \%$ of 40 patients with $\alpha \geq 66.3$ (Figure 2.4 ). $\mathrm{K}$ value is significantly associated with mortality

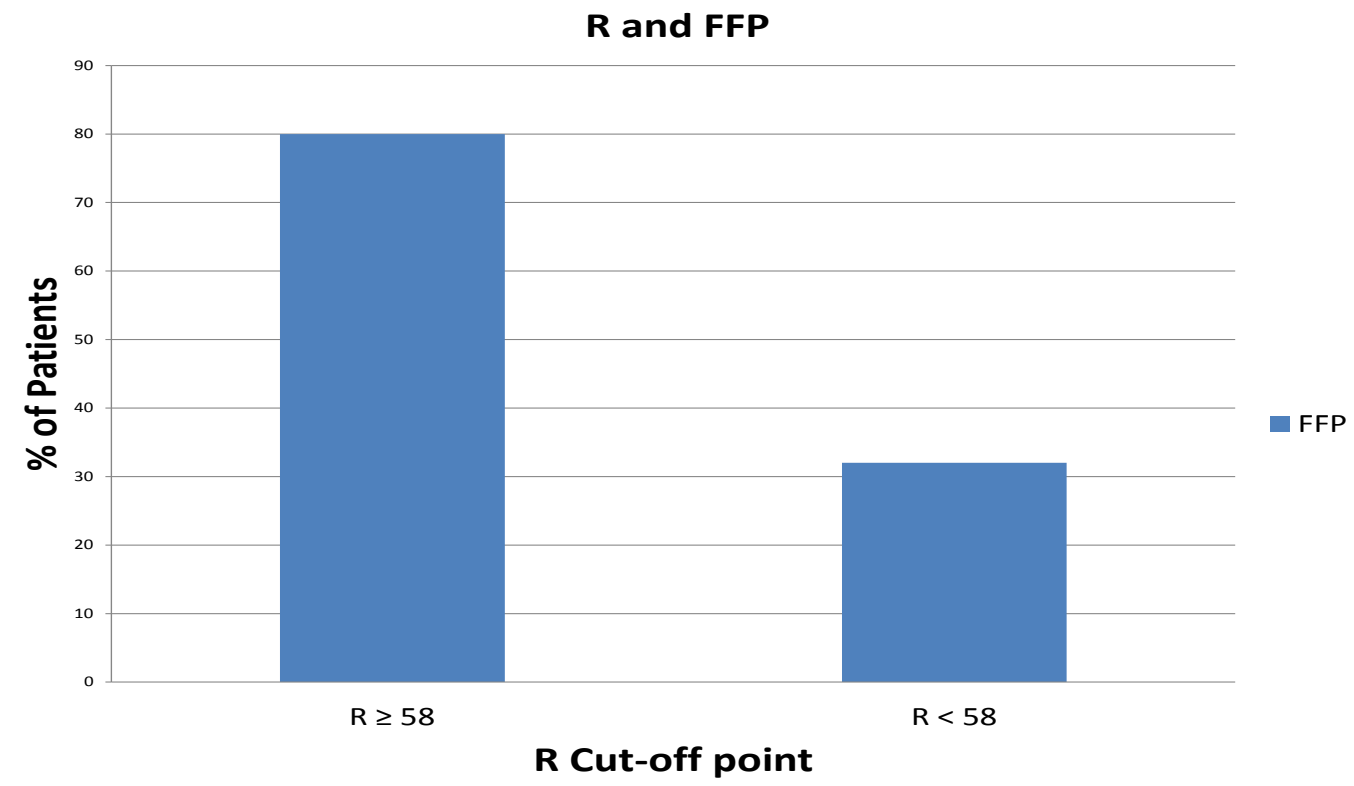

Figure 2.3: Correlation of R Cut-off Point with FFP Transfusion

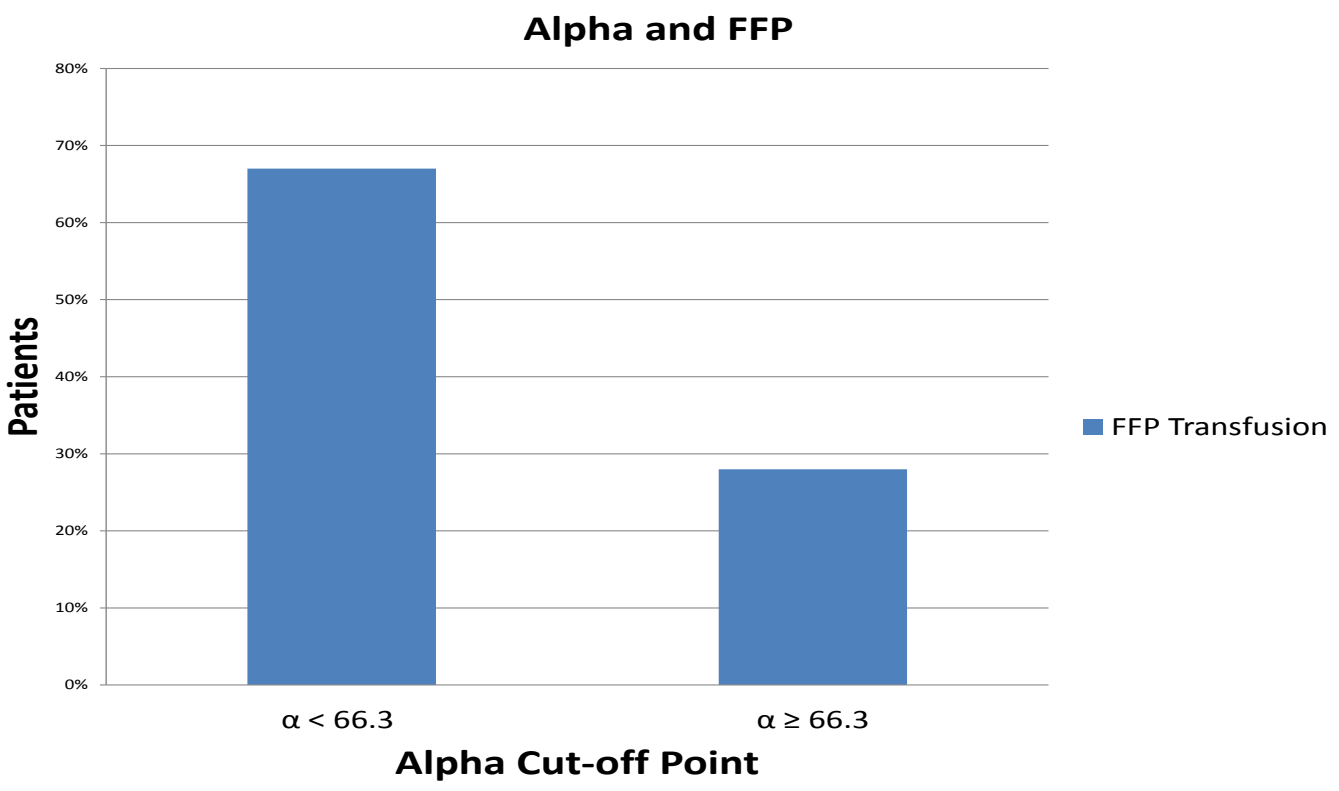

Figure 2.4: Correlation of Alpha Cut-off Point with FFP Transfusion 
( $p=0.0118)$, hypotension $(p=0.0172)$, and FFP transfusion $(p=0.0154)$ (Figures 2.5, 2.6, $2.7)$.

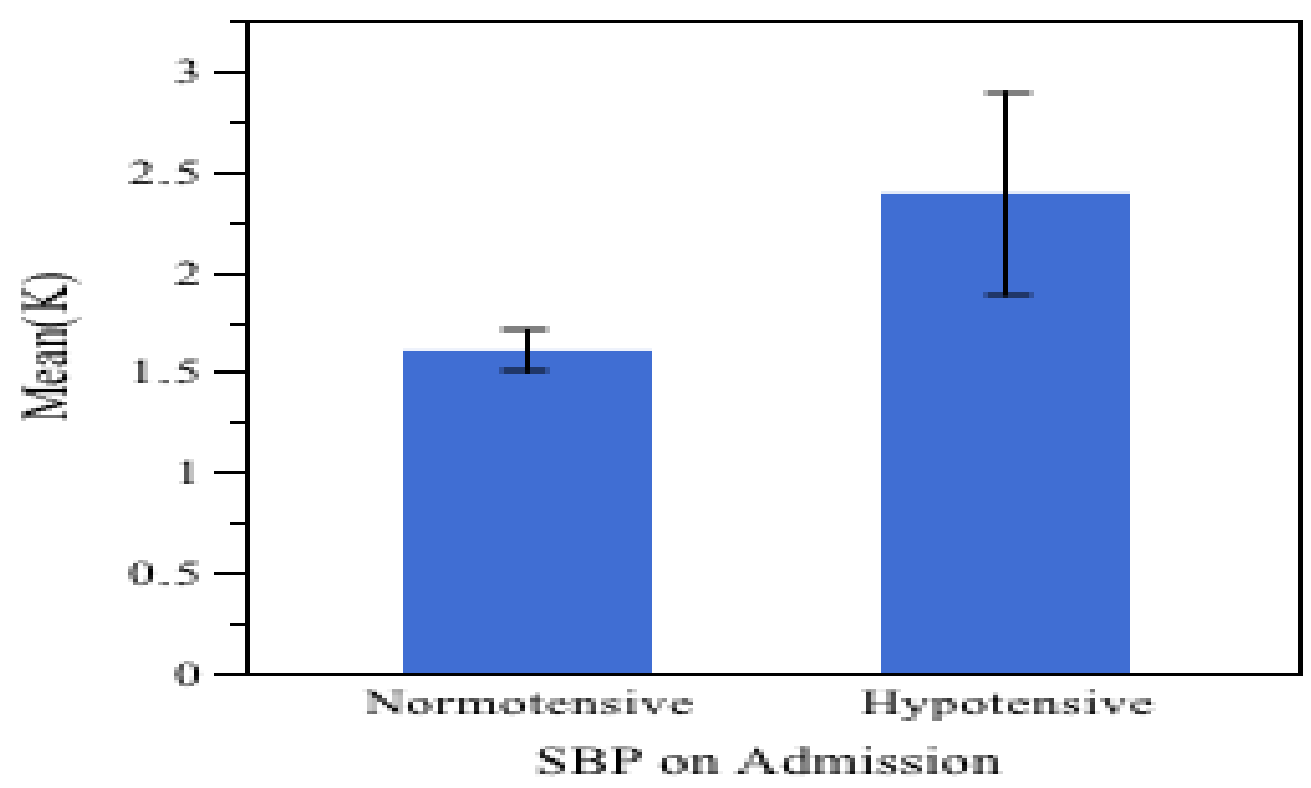

Figure 2.5: Correlation of K Measurement with Blood Pressure Status

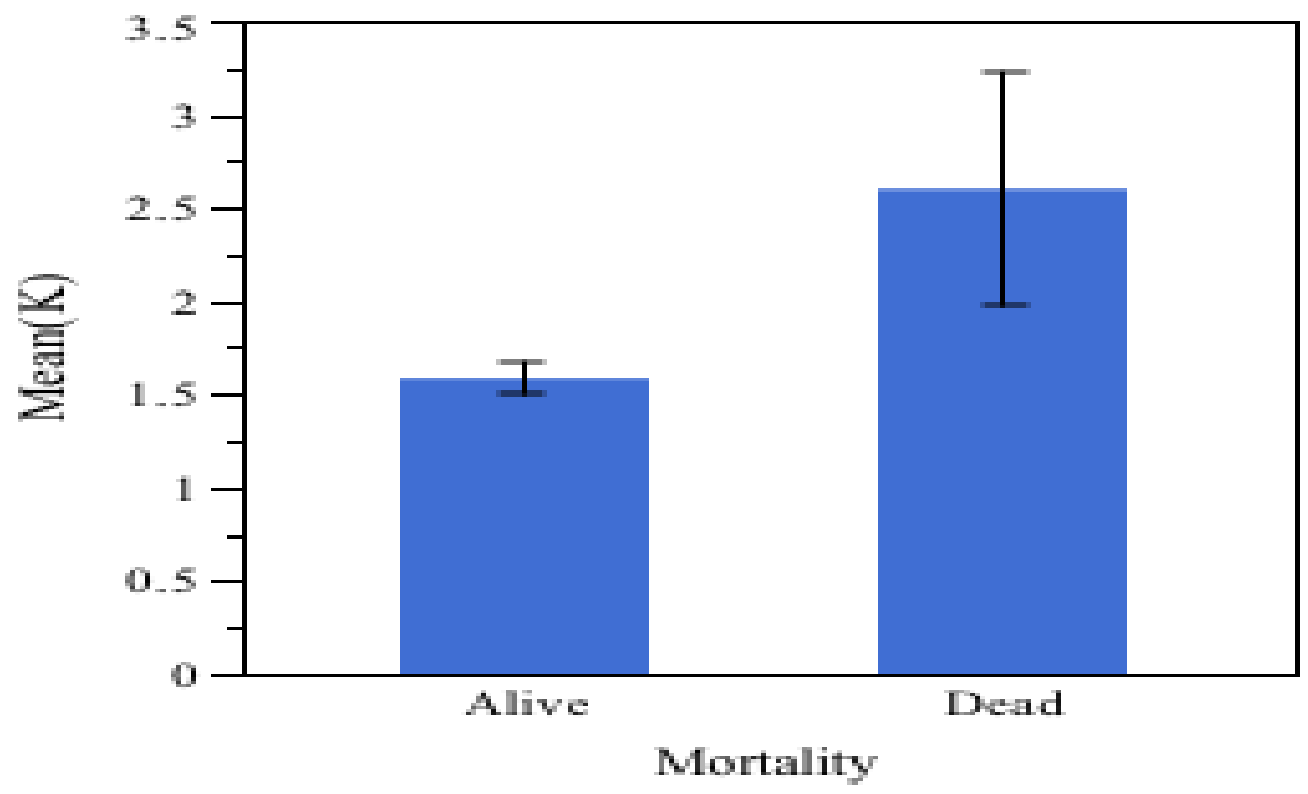

Figure 2.6: Correlation of K Measurement with Mortality Rate 


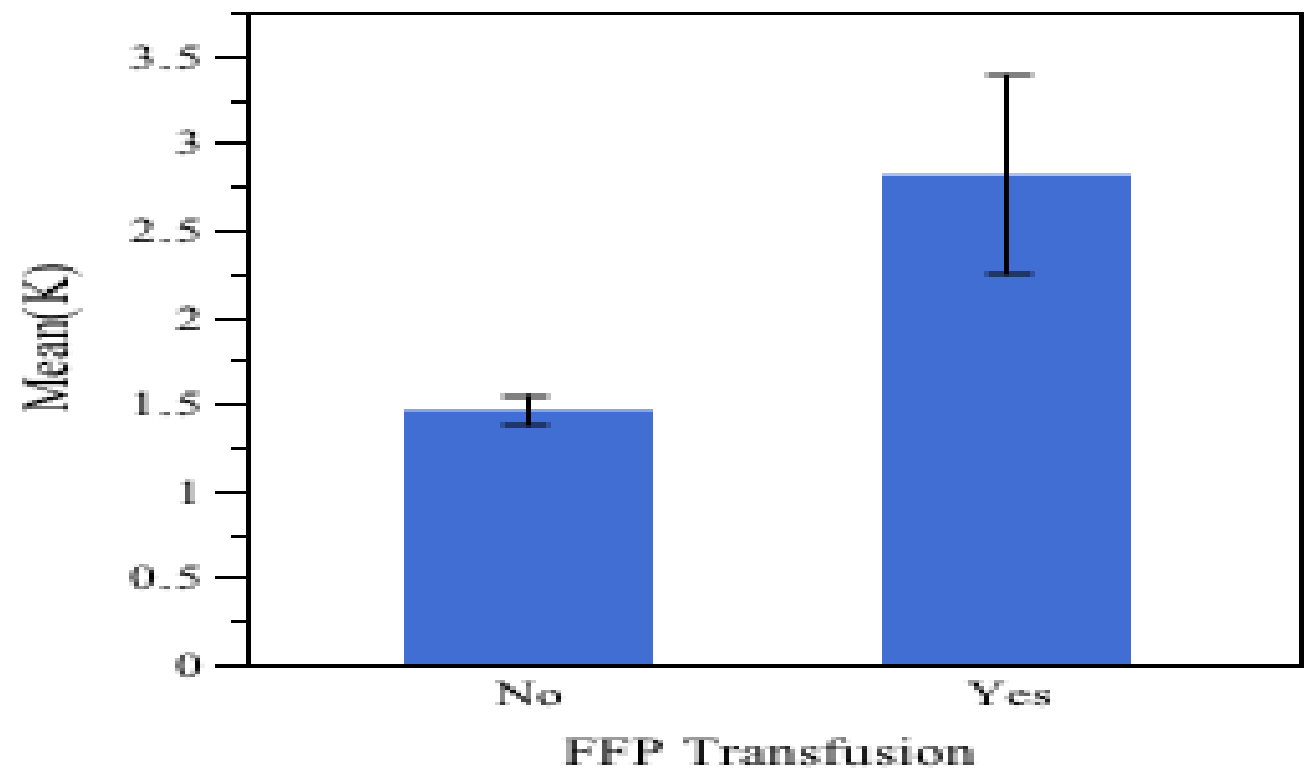

Figure 2.7: Correlation of K Measurement with FFP Transfusion

Decreased MA $(\mathrm{p}=0.0003)$, decreased K $(\mathrm{p}=0.0154)$, increased PT $(\mathrm{p}=0.0015)$, and increased INR $(\mathrm{p}=0.0014)$ were significantly associated with FFP transfusion. 


\section{Chapter 3}

\section{Discussion}

This study concludes that TEG parameters are a potentially useful means to rapidly diagnose coagulopathy and predict transfusion in trauma pts. Independently, presence of TBI does not cause a detectable coagulopathy on admission. MA is best single indicator for PRBC and/or FFP transfusion in trauma patients. TEG analysis is more efficient than the classical parameters in detecting patients who will need PRBC and FFP transfusion. Although, blood transfusion is a vital decision that can save patients lives especially in emergency and operating settings, there is no a quick and trustworthy test to assess in vivo the coagulation statuses of those patients[9]. Early transfusion of plasma and PRBC is a recommended step in the most massively bleeding and critically injured patients[10]. Nonetheless, a new study suggested that transfusing 6 platelet units for each 78 units of PRBC can improve the survival rates in massive transfusion patients[10]. Furthermore, giving extra cryoprecipitate units is not known to raise fibrinogen and von Willebrands factor or carry any benefits[10]. TEG can provide a foundation to develop a transfusion protocol that uses patients' hemostatic statuses[9]. This study reported that when MA is less that 58.1 $\mathrm{mm}$, the majority of the patients need PRBC and/or FFP. MA represents the maximum clot strength and relates to the quality of fibrin and platelet interaction which depends on platelet number and/or function[11]. This explains the precision and ability of MA and the failure of CCT parameters, including platelets number, to interpret such needs. Even though some changes in other parameters, K, PT, and INR, correlated with FFP transfusion that did not show a specific cut-off point nor any association with PRBC transfusion. K-time 
(clot formation time) represents the level of clot firmness and clot formation kinetics[11]. Our study reported that low $\mathrm{K}$ is significantly associated with hypotension and high mortality rates (lower among those who are alive and have no hypotension). This is due to the fact that patients who are hypotensive are more prone to develop bleeding disorders and increased death rates[7]. Additionally, the majority of our patients sample did not need FFP transfusion until their $\mathrm{R}$ values reached [3][4]. This reflects the ability of $\mathrm{R}$ to exclude those patients who have the least likelihood to need FFP. Furthermore, $\alpha$ Angle value of $\leq 62.9$ was able to predict the need of the preponderance $(77 \%)$ of the sample for transfusion. This finding has been found by other study that showed when $\alpha$ Angle parameter was utilized it predicted $84 \%$ of the patients need for transfusion[12]. The results are supported by previous similar studies that showed that TEG is a valuable technique to monitor the coagulation status of TBI patients. It can differentiate between the various causes of coagulopathy, and can provide appropriate guidance for blood, blood products and other intervention[9][13][14]. Moreover, Maegeles study revealed that TBI patients who develop coagulopathy have about 10 times higher chance of death than those patients who do not suffer from coagulopathy[8]. Additionally, Plotkins et al. study stated that PT and PTT are unable to determine which step of the clotting flow is affected in coagulopathic patients[9]. However, employing TEG can provide a more precise diagnosis of clotting dysfunctional disorders[9]. The CCT (INR and PT) showed only a significant association with the need for FFP transfusion, which has been also predicted by TEG parameters, MA and K. Furthermore, CCT did not show any advantage over TEG parameters on the rest of the studied variables, including the need for blood and other blood products transfusion, hypotension or the mortality rate. This can be explained by the limited abilities of CCT to detect the other hemostatic abnormalities that participate in bleeding tendency such as the efficiency of the platelets to develop stable clot when their numbers are within the normal range. We report that there is no difference between TBI and NTBI groups in terms of coagulation status that has been found by another study that stated that in order to develop TBI early coagulopathy, TBI patients should have a tissue injury and tissue hypoperfusion[5][7]. Additionally, according to Talvings et al. study, the published rates of coagulopathy in brain traumatic patients is not in agreement and shows very wide ranges (8\%-86\%).9 Furthermore, Genets et al. study concluded that 
there were no significant biomarker differences between TBI and non-TBI patients, and the coagulopathy status does not depend on the site of injury but rather on its severity[15]. In trauma settings, the main goal of damage control resuscitation (DCR) is to avoid the progress of the lethal triad of coagulopathy, metabolic acidosis, and hypothermia or to reduce the required time for the appropriate intervention once the triad begins to evolve[16]. Tailored intervention to treat coagulopathy has not been well defined; it has been considered only as a result of resuscitation, hemodilution, and hypothermia, and partially attributed to blood banking procedures delay[17]. DCR intraoperatively by blood products has showed a decent improvement in survival by adopting a rapid correction of trauma-induced coagulopathy in military and civilian injured patients[18]. Based on the fact that early development of coagulopathy in trauma patients, aggressive fluid replacement may lead to reperfusion injury, and massive transfusions are correlated with a high mortality rate, the most seriously injured patients may benefit from a resuscitation approach depends on the accurate timing and assessment of their coagulation statuses[19]. The current practice in patients who need massive transfusion is to give fresh frozen plasma, packed red blood cells, and platelets in a 1:1:1 ratio[20]. This approach is theoretically eye-catching; however, it has been built on only two retrospective observational studies that revealed improvement in the survival rate with this regimen. Thus, more rigorous studies are demanded before firm guidelines are recommended particularly in the civilian settings[20]. Based on the current and previous work that evaluated TEG effectiveness to assess the coagulation status, this study proposes using TEG to assess the coagulation statuses of the trauma patients and predict their accurate needs for transfusions[12][4][13]. This study has a number of strengths. First, this study was analyzed over more participants (142) as compared to other studies such as Park et al.[4] (78), Plotkin et al.[9] (44), and Cohen et al.[7] (39). Second, this is the first study to our knowledge comparing TBI with NTBI in terms of TEG and CCT parameters. Third, sending the TEG sample from the trauma-bay, within 15 minutes after arrival, leads to highly reliable results. This is because the samples are taken before any blood or blood products transfusion. This matches the recommended guidelines for standard coagulation measures[4]. There were a number of constraints in the study. First, it was a retrospective and a single center study. Second, some classical coagulation test results are missing. Third, 
transferred patients from the other facilities could spend a long time before they get TEG samples drawn and/or received blood or blood products; however all of them came through the ED as a trauma activation, thus they still have the same timeline. Fourth, fibrinogen, which is one of the most sensitive CCT, was not collected for the majority of the patients; however, numerous trauma centers do not depend on this test, since results are commonly not available for several hours[12]. Future research should be prospective where more than one TEG test can be run on each patient, complications can be recorded, and be compared to the results of the tests (TEG or CCT) which can predict the clinical correlation. Multicentric study can eliminate any subjective bias, use the same protocol in all facilities, and avoid the above stated limitations. Also, we suggest further investigation of TEGs cut-off points that showed significant results in this study. 


\section{References}

[1] Kaur, Paramjit, Sabita Basu, Gagandeep Kaur, and Ravneet Kaur., "Transfusion protocol in trauma," Journal of Emergencies, Trauma and Shock, vol. 4, pp. $103-108$, 2011.

[2] Shaz, Beth H, Christopher J. Dente, Robert S. Harris, Jana B. MacLeod, and Christopher D. Hillyer, "Transfusion management of trauma patients," Anesthesia and Analgesia., vol. 108, pp. 1760-1768, 2009.

[3] Sankarankutty, Ajith, Bartolomeu Nascimento, Luis Teodoro da Luz, and Sandro Rizoli, "Teg and rotem in trauma: similar test but different results?," World Journal of Emergency Surgery, vol. 7, pp. 1-8, 2012.

[4] Myung S Park, Wenjun Z. Martini, Michael A. Dubick, Jose Salinas, Saulius Butenas, Bijan S. Kheirabadi, and Anthony E. Pusateri et al, "Thromboelastography as a better indicator of postinjury hypercoagulable state than prothrombin time or activated partial thromboplastin time," The Journal of trauma, vol. 67, pp. 266-276, 2009.

[5] Talving, Peep, Rodd Benfield, Pantelis Hadjizacharia, Kenji Inaba, Linda S. Chan, and Demetrios Demetriades, "Thromboelastography as a better indicator of postinjury hypercoagulable state than prothrombin time or activated partial thromboplastin time," The Journal of Trauma and Acute Care Surgery, vol. 66, pp. 55-62, 2009.

[6] Stein, Sherman C, and Douglas H. Smith, "Coagulopathy in traumatic brain injury," Neurocritical Care, vol. 1, pp. 479-488, 2004.

[7] Cohen, Mitchell Jay, Karim Brohi, Michael T. Ganter, Geoffrey T. Manley, Robert C. Mackersie, and Jean-Franois Pittet, "Early coagulopathy after traumatic brain injury: the role of hypoperfusion and the protein c pathway," The Journal of Trauma and Acute Care Surgery, vol. 63, pp. 1254-1262, 2007.

[8] Maegele Marc, "aand treatment options," Transfusion, vol. 53, pp. 28S-37S, 2013.

[9] Plotkin Amy J, Charles E. Wade, Kimberly A. Smith Donald H. Jenkins, Jody C. Noe, Myung S. Park, Jeremy G. Perkins, and John B. Holcomb, "A reduction in clot formation rate and strength assessed by thrombelastography is indicative of transfusion requirements in patients with penetrating injuries," The Journal of Trauma and Acute Care Surgery, vol. 64, pp. S64-S68, 2008. 
[10] Ketchum, Lloyd, John R. Hess, and Seppo Hiippala, "Indications for early fresh frozen plasma, cryoprecipitate and platelet transfusion in trauma," The Journal of Trauma and Acute Care Surgery, vol. 60, pp. S51-S58, 2006.

[11] Curry, Andy NG, and JM Tom Pierce, "Conventional and near-patient tests of coagulation," Continuing Education in Anaesthesia, Critical Care and Pain, vol. 7, pp. 45-50, 2007.

[12] Jeger, Victor, Sandra Willi, Tun Liu, Daniel D. Yeh, Marc De Moya, Heinz Zimmermann, and Aristomenis K. Exadaktylos., "The rapid teg alpha angle may be a sensitive predictor of transfusion in moderately injured blunt trauma patients," The Scientific World Journal, pp. 1-7.

[13] da Luz, Luis Teodoro, Bartolomeu Nascimento, and Sandro Rizoli, "Thrombelastography: practical considerations on its clinical use in trauma resuscitation," Scandinavian journal of trauma, resuscitation and emergency medicine, vol. 21, pp. 1-8, 2013.

[14] Chiumello D M., Cressoni C, De Grandis L. Landi, and M. Racagni, "24th international symposium on intensive care and emergency medicine," Critical Care, vol. 8, pp. 1-2, 2004.

[15] Gent, Gustav Folmer, Par Ingemar Johansson, Martin Abild Stengaard Meyer, Sacha Slbeck, Anne Marie Srensen, Claus Falck Larsen, Karen Lise Welling, Nis Agerlin Windelov, Lars S. Rasmussen, and Sisse Rye Ostrowski., "Trauma-induced coagulopathy: Standard coagulation tests, biomarkers of coagulopathy, and endothelial damage in patients with traumatic brain injury," Journal of neurotrauma, vol. 15, pp. 301-306, 2012.

[16] Brooks, Adam J, and Bryan A. Cotton, "Damage control resuscitation," In Ryan's Ballistic Trauma, pp. 251-261.

[17] Holcomb, John B, Don Jenkins, Peter Rhee, Jay Johannigman, Peter Mahoney, Sumeru Mehta, and E. Darrin Cox et al., "Damage control resuscitation: directly addressing the early coagulopathy of trauma," The Journal of Trauma and Acute Care Surgery, vol. 62, pp. 307-310, 2007.

[18] Duchesne, Juan C, Katerina Kimonis, Alan B. Marr, Kelly V. Rennie, Georgia Wahl, Joel E. Wells, and Tareq M. Islam et al, "Damage control resuscitation in combination with damage control laparotomy: a survival advantage," The Journal of Trauma and Acute Care Surgery, vol. 69, pp. 46-52, 2010.

[19] John B. Holcomb, "Damage control resuscitation," J Trauma, vol. 62, pp. S36 S37, 2007.

[20] Jansen J. R, Thomas. M, Loudon, and A Brooks, "Damage control resuscitation for patients with major trauma," BMJ, vol. 1778, pp. 1436 1440, 2009. 